\title{
Passive Viewpoints in a Collaborative Immersive Environment
}

\author{
Sarah Coburn, Lisa Rebenitsch, and Charles Owen \\ Computer Science \& Engineering, Michigan State University, East Lansing, MI, USA \\ \{coburnsa, rebenits, cbowen\} @cse.msu.edu
}

\begin{abstract}
Widespread acceptance of virtual reality has been partially handicapped by the inability of current systems to accommodate multiple viewpoints, thereby limiting their appeal for collaborative applications. We are exploring the ability to utilize passive, untracked participants in a powerwall environment. These participants see the same image as the active, immersive participant. This does present the passive user with a varying viewpoint that does not correspond to their current position. We demonstrate the impact this will have on the perceived image and show that human psychology is actually well adapted to compensating for what, on the surface, would seem to be a very drastic distortion. We present some initial guidelines for system design that minimize the negative impact of passive participation, allowing two or more collaborative participants. We then outline future experimentation to measure user compensation for these distorted viewpoints.
\end{abstract}

Keywords: virtual reality, passive participation, immersion, perception.

\section{Introduction}

Many immersive environments are unavailable to the general public because they are limited to large rooms and they require a tremendous budget (sometimes even exceeding \$1 million [1]). One solution is the one-walled VR environment (the "powerwall"), which can be implemented with a single screen and projector, and can use a number of different widely available tracking systems.

Many existing virtual reality (VR) systems only allow one or two users. When VR demos or experiments are performed, extra shutter or polarized glasses are often used for additional viewers to sit in and watch the world in the role of passive participants in the virtual experience. With this in mind, can we create collaborative VR systems that can actually involve these passive participants, allowing them to experience and interact with the virtual environment, alongside an active, tracked participant? A common obstacle to the adoption of VR is that so many systems are fundamentally solitary experiences. Some hardware approaches allow for even up to six active users, but these approaches are expensive, difficult to set up and calibrate, and not as available as basic single-user systems. In order to display individual perspectives, these systems often add multiple projectors and polarized images in order to project images to the left and right eyes of each participant [2][3][4]. These systems show promise 
for the next generation of VR collaboration, but the resulting cost and computation power makes it less accessible to the general public, as well as difficult to extend to even more participants. As a result, we are examining the design of systems that will allow multiple passive users to participate with an active user in a VR environment, possibility with the ability to pass the active experience among users as necessary, so as to support collaborative applications. This paper addresses some of the physical and psychological issues associated with the participation of passive users and explores some initial design guidelines to tailor applications for passive participation.

One of the greatest challenges in designing a collaborative virtual environment is making sure that all participants can accurately perceive the virtual world. In simpler virtual environments, if multiple participants view the world, virtual objects are typically presented based on a single center of projection from an oblique angle. To design a better multi-user, one-walled VR system, the design must have knowledge of where the passive participants are, as well as what elements in the environment can be manipulated to counteract the perception challenges. Knowing the location of the user allows manipulation of the virtual world to actively adapt to passive participants, even when there is still only a solitary center of projection. In addition, the VR system can take advantage of the psychological ability to compensate for distortions.

In this article, we explore the appearance and perception of the virtual experience as seen by a passive participant. Section 2 presents a mathematical model for the image as seen by passive participants, regardless of their relative position to the screen or the active participant. Section 3 explores the psychological and physiological components of a passive participant's perception of the virtual world. Finally, Section 4 establishes some leading guidelines for the design of virtual environments that take advantage of these elements.

\section{Mathematical Basis}

One of the most basic VR systems is the powerwall, which uses a rear projection stereo display or a large 3D monitor to provide a virtual experience for a single user with 3D glasses and head tracking. Fig.1 illustrates the basic projection model for a powerwall. It is assumed that the world coordinate system origin is centered on the screen with the $\mathrm{Y}$ axis up and the $\mathrm{X}$ axis to the right and we assume a right-hand coordinate system. In the figure, the point $p_{a}\left(x_{a}, y_{a}, z_{a}\right)$ represents the active participant. This participant is tracked and the basis for the projection. The point $p_{b}$ $\left(x_{b}, y_{b}, z_{b}\right)$ represents a passive participant. This participant is untracked and sees the same image as the active participant. The point $v(x, y, z)$ represents a point in the virtual space subject to projection. The point $p\left(x_{p}, y_{p}, z_{p}\right)$ represents the projected point on the screen. Projection is accomplished by creating a projector, a ray starting at the center of projection and pointing at the virtual point, and computing the intersection of that ray with the virtual projection surface. 


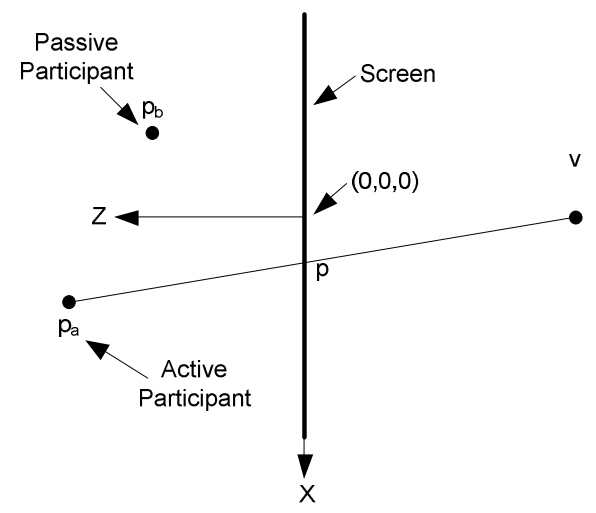

Fig. 1. Model for screen-based VR projection

Given this orientation, projection is implemented in real systems by changing the origin to the active participant viewpoint and multiplying by the ratio of the distance of the projection screen (focal length) and the z-axis distance to the point. (Real-world systems also include a mapping from the virtual projection screen to pixels on the real projection screen, but that linear mapping does not affect the results in this paper and is, therefore, ignored). The basic equations for projection are:

$$
\begin{aligned}
& x_{p}=\left(x-x_{a}\right)\left(\frac{-z_{a}}{z-z_{a}}\right) \\
& y_{p}=\left(y-y_{a}\right)\left(\frac{-z_{a}}{z-z_{a}}\right)
\end{aligned}
$$

Since projection for $\mathrm{x}$ and $\mathrm{y}$ is symmetrical, only the $\mathrm{x}$ component will be indicated in future equations. The y component can be determined through simple substitutions. All of the equations in this section apply for virtual points on either side of the screen.

\subsection{Depth}

The passive participant would ideally view the world projected properly for them. However, they instead see the world as projected for that active participant. The first question is: what is the equivalent mapping between the two? The multiplicative factor from Equation 1:

$$
\left(\frac{-z_{a}}{z-z_{a}}\right)
$$

determines the foreshortening due to depth. Foreshortening is the tendency of objects under perspective projection to appear smaller or larger due to their distance from the viewer. The foreshortening seen by the passive participant will be different if the participant is not at the same distance from the screen as the active participant. Intuitively, if the passive participant is half the distance to the screen, the amount of foreshortening will be doubled. This is the same as if the depth of the virtual space was scaled by a factor of 0.5 , making objects appear to be half as far away as they are. 
We can illustrate this by scaling the $\mathrm{z}$ values for the passive participant's foreshortening term by $z_{p} / z_{a}$ :

$$
\frac{-z_{p}}{z_{p} \frac{z_{a}}{z_{a}}-z_{p}}=\frac{-z_{p} z_{a}}{z z_{p}-z_{p} z_{a}}=\frac{-z_{a}}{z-z_{a}}
$$

What this means is that a passive participant moving closer to the screen will see a world that appears scaled in the $\mathrm{z}$ dimension.

\subsection{Position}

Given the solution for the change of depth for perceived point, we can now examine the $x, y$ components of the perceived point for the passive participant. Figure 2 illustrates the geometry of the problem. The point $v$ is projected to point $p$ on the screen for the active participant. The passive participant sees that point at point $p$ as some point along the projector from $p_{b}$ through point $p$ to the point $v^{\prime}$ as it appears to that participant. We know the depth of $v^{\prime}$ is $z z_{p} / z_{a}$ due to Equation 4.

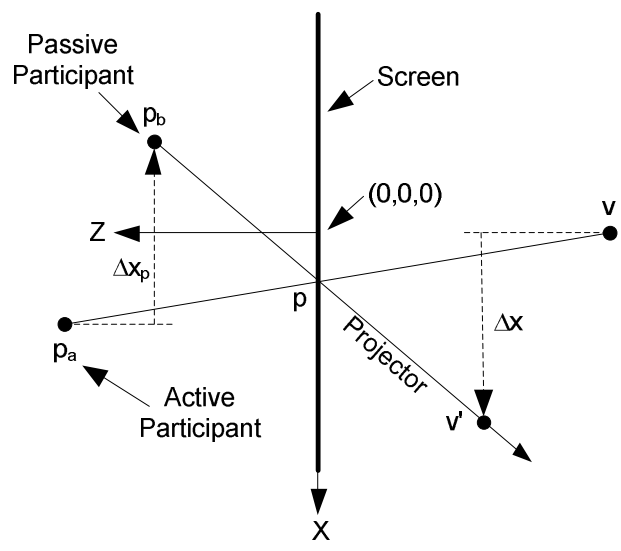

Fig. 2. Geometry of virtual point displacement

The geometry is proportional between the two sides. If the passive participant position is offset from the active participant by $\Delta \mathrm{x}_{\mathrm{p}}=\mathrm{x}_{\mathrm{p}}-\mathrm{x}_{\mathrm{a}}$, the perceived position of the point will be offset by the negative of that value scaled by the ratio of the point depth divided by the view distance for the active participant:

$$
x^{\prime}=x+\left(x_{p}-x_{a}\right) \frac{z}{z_{a}}
$$

Effectively, this imparts a skew on the virtual world. The amount of the skew is determined by the difference between the positions of the active and passive participants and is based on depth. Given these solutions, the passive participant will perceive the point $(x, y, z)$ at this position: 


$$
\begin{gathered}
x^{\prime}=x+\left(x_{p}-x_{a}\right) \frac{z}{z_{a}} \\
y^{\prime}=y+\left(y_{p}-y_{a}\right) \frac{z}{z_{a}} \\
z^{\prime}=z \frac{z_{p}}{z_{a}}
\end{gathered}
$$

\subsection{Stereo}

The depth scaling also holds for stereo disparity, assuming the interpupilary distance (IPD) and eye orientations for the active and passive participants are the same. This relationship is illustrated in Figure 3.

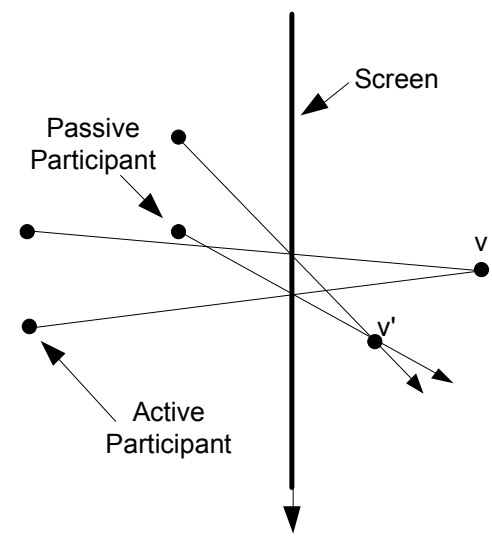

Fig. 3. Depth scaling due to stereo disparity

As many stereo installations assume only head position tracking and fixed IPD values, the stereo depth scaling will correspond to that due to this varying participant screen distances. If the IPD distance or orientation is allowed to vary, there will be a divergence, which can be confusing.

\section{Psychological Considerations}

As illustrated in Section 2, the distortions resulting from a non-primary viewpoint present the passive observer with an image of object that are skewed and distorted, rather than the correct proportions. However, there is evidence for psychological compensation for this distortion, allowing observers to correctly perceive object proportions, orientations, and relative positions in a variety of visual mediums. From paintings to photographs, people are actually quite accustomed to viewing images from a point that does not correspond to the center of projection. 


\subsection{Potential Psychological Complications}

Judging Object Orientations. One characteristic of a virtual scene is the orientation of the projected objects. If viewed differently by the individual participants, there is a potential confound of collaboration.

Goldstein [5,6] discussed three main pictorial perception attributes: perceived orientation (in relation to the observer), perceived spatial layout (of the objects in the image), and perceived projection (perception on the observer's retina). He determined that relative position of objects in an image (in the virtual space) was maintained, despite a variety of oblique viewing angles. However, he did find that perceived orientation was not maintained as the difference in angle from the center of projection increased. As a result, Goldstein identified the differential rotation effect (DRE), where objects in a $2 \mathrm{D}$ image pointing to the observer (at an angle near $90^{\circ}$ ) appear to move faster than objects farther away when the observer moves relative to the image.

One popular virtual collaboration medium is the interactive tabletop. Multiple users interact with a single table, which has virtual objects presented from a common viewpoint. Hancock et al [7] analyzed user perception of orientation of objects projected on a tabletop device, and found that users had a more difficult time judging object orientation when the COP was farther away from their own viewpoint. They determined that a central, neutral COP helped to minimize discrepancies between user perceptions. This study also found that using an orthographic projection (such as found in blueprint drawings to maintain correct object proportions) aided the users in identifying the orientation of objects, though this projection does eliminate foreshortening, an important depth cue. However, though a tabletop design often requires a $360^{\circ}$ of potential user viewpoints, CAVE environments (both single and multiple screen displays) naturally limit the range of these viewpoints. This suggests that a neutral viewpoint might be more effective in a CAVE environment than the study found for the tabletop.

Cybersickness. A common element in virtual systems is cybersickness, a form of motion sickness associated with virtual environments. While the exact causes of cybersickness are still under investigation, it is known to be common in situations where the camera viewpoint moves independently of movement from passive observers.

Cybersickness symptoms are prevalent in most VR environments and can impact from $\sim 30 \%$ [8] to $~ 80 \%$ [9] of participants. Displaying imagery that is not controlled by the observer generally increases symptoms. Swaying imagery is often used in cybersickness research to invoke symptoms. Chen et al. [8] and Dong and Stoffregen [10] demonstrated this effect with experiments where participants were "drivers" or "passengers" in a virtual car. Drivers were given a tracked viewpoint and control of their environment and the resulting video was recorded. This video was then displayed to a passenger. Not surprisingly, passengers had more cybersickness symptoms than drivers. Passengers also had high amounts of body movements, indicating a strong involvement (and conflict) with the movement in the recording.

In both of these situations, participants who suffered cybersickness symptoms were not in control of the camera viewpoint. If a collaborative environment must share a common viewpoint, the design should consider ways to minimize the cybersickness effects that will result. Another major source of cybersickness symptoms is when visual and kinetic information conflict. This makes sharing a common viewpoint difficult: passive viewers are not in control of changes to the perspective. 


\subsection{Compensation for Distortion}

In order to determine what characteristics of the virtual scene can be or even need to be modified to help accentuate depth and spatial cues, we must first explore what the brain will use in order to compensate for missing stimuli.

Gestalt psychology here argued that the mind has the ability to organize visual stimuli, and is structured and operates in such a way to make this possible. As opposed to the empirical belief (which says we only learn through experience with the world), Gestalt psychology says that the "brain uses organizational principles to interpret visual appearance" [11].

Because of this ability of the mind to natively organize incoming visual stimuli, the mind can still form a cognitive model of the object in a distorted picture. Since it is not purely empirical, the distortion does not negate the ability to form and access mental models.

Spatial Cognition. Spatial cognition is the ability of the brain to acquire, organize, utilize, and revise knowledge about spatial environments [12]. Knowledge of this cognitive ability is especially helpful in creating virtual environments. If certain characteristics of the system hamper the participant's ability to correctly perceive the virtual world, knowledge of the mind's organizational power can help compensate for the shortcomings.

The neuroscientist David Marr identified three stages of visual processing, including what he coined the "3D sketch," when the mind visualizes the world in a mental 3D model [13]. Intelligent perception allows the mind to make cognitive observations about this 3D data. One theory of the brain's ability to categorize visual information is that rather than purely learning from experience (empirical view) the brain actively organizes incoming visual sensory data into mental models. Gestalt psychology established this theory, and began to identify the specific characteristics that the brain uses to categorize visual data.

People routinely view 2D imagery from a wide range of angles and have little difficulty with correctly interpreting the data in the image. Vishwanath et al. [14] suggested that 2D pictures look correct when viewed from the wrong angle not by geometric data inside the picture itself, but from an estimate of local surface orientation. When binocular vision was available, participants were able to correctly judge object characteristics invariant of their viewing angle. Instead of judging object characteristics by observing the objects within the image, participants instead used cues from the local slant of the image itself to correct perspective distortion from viewing the picture obliquely.

Spatial and Depth Cues. Despite the known variations of object characteristics between different viewpoints, there are many other cues that the human brain will use to determine spatial characteristics. As discussed by Christou and Parker [11], there are several pictorial cues that allow 2D images to represent and simulate 3D objects. These cues can be manipulated by a virtual system, and can simulate natural objects by imitating perspective and depth. Most of these cues can be emphasized to compensate for any existing distortions: 
Shading: Shading and gradients can easily be used to make closer elements brighter and clearer, while more distant objects (or the distant parts of an object) will have darker or dimmer shading, depending on the lighting in the scene.

Color Fading: Similar to shading, color fades when farther away, so closer objects will have higher color saturation. Atmospheric effects also add to this: objects farther in the distance are often dimmed due to water, dust, or other particles in the air.

Interposition: Objects closer to the observers will occlude objects farther away.

Shadows: Shadows from the objects in the world on a flat surface can help establish their locations (both relative to each other and relative to the world).

Binocular Cues: Unlike 2D pictures, 3D virtual environments show images to both eyes, which allow stereoscopic cues to show depth. The visual system uses the slight differences in the two pictures (that are a result of the interpupilary distance) to gain significant information about spatial depth and location of objects in a scene.

Object Shapes: Another example of compensation for distorted objects by the visual system is when rigid and recognizable shapes are presented. Perkins [15] found that participants would compensate for oblique views of rectangular solids, and even when the judgments were inaccurate, they were only inaccurate by a small amount. If mental models are formed for commonly encountered objects, then it will be easier to match an image of such an object to the correct mental model, even if distorted.

\section{$4 \quad$ Guidelines for Design}

The main reason for examining the issues related to passive participants in a one-wall cave setting is the ability to better utilize these environments in groups. Traditionally, virtual reality systems have been solitary experiences. Complex hardware solutions have often allowed for two participants, but a group setting with a dozen users has been considered impractical other than in large theatrical settings or where the tracking is severely limited. We seek to exploit the idea of passive participants to create group VR applications and are exploring both the impact on passive participants and the transfer of active status among participants.

One major influence on design is a factor we are calling leverage. As seen in Equation 6, the amount of offset (skew) of the perceived point is scaled by the ratio $z / z_{a}$. As the distance from the screen increases, the offset also increases. As an example, if the active participant moves to the right $10 \mathrm{~cm}$, an object close in depth to the screen ( $\mathrm{z}$ values near zero) will move very little. However, an object far from the screen, such as an object in the far distance, will move a very large amount.

Given this concept of leverage and the psychological concepts in Section 3, we are proposing several design guidelines for passive participant powerwall systems. These guidelines are intended to be used when distortions affect passive participation, to raise the overall utility of the system.

Guideline 1: Keep as much content at a depth near the screen as possible. This is the most obvious design guideline, since it minimizes the amount of leverage, and it keeps objects within interaction reach. 
Guideline 2: When possible, the active participant should be close to the screen. An active participant close to the screen will minimize the ratio $z / z_{a}$ in Equation 6 for user interface elements. Similarly, the active participant should remain closer to the center of the screen, so that the controlling viewpoint is the average of the possible passive participant viewpoints. This causes fewer overall discrepancies between viewpoints.

Guideline 3: Passive participates should be farther from the screen. Decreasing the angle of sight lines between the active and passive participant decreases the relative discrepancy in orientations. Moving users back limits the range of angles for these users, thus decreasing orientation confusion due to the differential rotation effect. In a setting where the active participation passes from user to user, this assignment may be based on physical position with a "move up to take control" type of approach.

Guideline 4: Avoid backgrounds or fix them in place. The problem with backgrounds is that they are naturally the farthest from the screen and, therefore, the most subject to leverage. Since they are often far in distance, users do not expect them to move that much and appear to be comfortable with this solution in preliminary experiments. This guideline also has the potential to minimize cybersickness effects that result from a moving background.

Guideline 5: Virtual objects should be familiar to users. Well-known or familiar objects appeal to long-established mental models. Because of this object recognition, users are better able to compensate for distortions in virtual objects. Overall, using familiar objects will help connect a virtual object to the user's sense of its physical presence in the real world. However, if novel objects are used, utilize Guideline 6.

Guideline 6: When familiar objects cannot be used to satisfy Guideline 5, emphasize pictorial cues such as shading, color fading, and atmospheric effects. This can help compensate for the lack of familiarity, allowing for novel virtual objects.

\section{Future Work}

We are currently examining a new modality for virtual reality environments that is designed to optimize the experience for a group of users, rather than a single participant. Our first focus is discovering and alleviating the problems passive observers experience in immersive environments, such as perception conflicts for passive observers, as well as triggers for cybersickness. This work will then move beyond alleviating these problems, and leverage psychological and physiological compensatory mechanisms in order to deliver a better group interface for virtual environments. We are also exploring enhancements for a group virtual environment, such as efficient ways to transition between active and passive participants, creating a more satisfying collaborative, immersive, environment. To demonstrate the guidelines presented in this paper, we are designing an experiment that will demonstrate the ability of a user to sufficiently judge object characteristics in a powerwall system, despite being removed from the primary viewpoint. 


\section{References}

1. Ramsey, D.: 3D Virtual Reality Environment Developed at UC San Diego Helps Scientists Innovate (2009), http://ucsdnews.ucsd. edu/newsrel/general/09083DVirtualReality.asp (accessed January 20, 2013)

2. Agrawala, M., Beers, A.C., McDowall, I., Fröhlich, B., Bolas, M., Hanrahan, P.: The Twouser Responsive Workbench: Support for Collaboration through Individual Views of a Shared Space. In: Proceedings of the 24th Annual Conference on Computer Graphics and Interactive Techniques (SIGGRAPH 1997), pp. 327-332. ACM Press/Addison-Wesley Publishing Co., New York (1997)

3. Fröhlich, B., Blach, R., Stefani, O., Hochstrate, J., Hoffmann, J., Klüger, K., Bues, M.: Implementing Multi-Viewer Stereo Displays. In: Proceedings: WSCG (Full Papers), pp. 139-146 (2005)

4. Kulik, A., Kunert, A., Beck, S., Reichel, R., Blach, R., Zink, A., Fröhlich, B.: C1x6: A Stereoscopic Six-User Display for Co-located Collaboration in Shared Virtual Environments. In: Proceedings of the 2011 SIGGRAPH Asia Conference, SA 2011 (2011)

5. Goldstein, E.B.: Perceived Orientation, Spatial Layout, and the Geometry of Pictures. In: Ellis, S.R., Kaiser, M.K., Gunwald, A.J. (eds.) Pictorial Communication in Virtual and Real Environments, ch. 31, pp. 480-485. Taylor \& Francis Inc., Bristol (1991)

6. Goldstein, E.B.: Spatial Layout, Orientation Relative to the Observer, and Perceived Projection in Pictures Viewed at an Angle. Journal of Experimental Psychology: Human Perception Performance 13, 256-266 (1987)

7. Hancock, M., Nacenta, M., Gutwin, C., Carpendale, S.: The Effects of Changing Projection Geometry on the Interpretation of 3D Orientation on Tabletops. In: ACM International Conference on Interactive Tabletops and Surfaces, New York, pp. 157-164 (2009)

8. Chen, Y., Dong, X., Hagstrom, J., Stoffregen, T.A.: Control of a Virtual Ambulation Influences Body Movement and Motion Sickness. In: BIO Web of Conferences, vol. 1(16) (2001)

9. Kim, Y.Y., Kim, H.J., Kim, E.N., Ko, H.D., Kim, H.: Characteristic Changes in the Psychological Components of Cybersickness. Psychophysiology 42(5), 616-625 (2005)

10. Dong, X., Stoffregen, T.A.: Postural Activity and Motion Sickness Among Drivers and Passengers in a Console Video Game. Proceedings of the Human Factors and Ergonomics Society Annual Meeting 54(18), 1340-1344 (2010)

11. Christou, C., Parker, A.: Visual Realism and Virtual Reality: A Psychological Perspective. In: Carr, K., England, R. (eds.) Simulated and Virtual Realities, ch. 3, pp. 53-84. Taylor \& Francis (1995)

12. Cognitive Systems. Spatial Cognition (2012), http://www. spatial-cognition. de (accessed January 19, 2013 )

13. Marr, D.C.: Vision: A Computational Investigation into the Human Representation and Processing of Visual Information. W.H. Freeman, New York (1983)

14. Vishwanath, D., Girshick, A.R., Banks, M.S.: Why Pictures Look Right When Viewed from the Wrong Place. Nature Neuroscience 8(10), 1401-1410 (2005)

15. Perkins, D.N.: Compensating for distortion in viewing pictures obliquely. Attention, Perception, \& Psychophysics 14(1), 13-18 (1973) 